\title{
Performance assessment of centrifuge dewatering unit using multivariate statistical approach: a case study of a centralized sludge treatment facility (CSTF) in Malacca, Malaysia
}

\begin{abstract}
The performance of the centrifuge dewatering unit in Sungai Udang centralized sludge treatment facility has been studied using multivariate statistical approach. The relationships between bio-solids production and 14 parameters were analyzed using principle component analysis (PCA) and multiple linear regression (MLR) analysis. PCA was used to simplify the complexity among variables affecting the production of bio-solids in the treatment facility. All varimax factor (VF) values obtained from the PCA were used as independent variables in MLR analysis. It was found that VF1 (wet sludge and mixed liquor suspended solids) and VF4 (polymer dosage) had significant linear relationships with bio-solids production, which accounted for $74.32 \%$ of variations in the bio-solids production. This approach could be used to precisely estimate the amount of sludge produced by the centrifuge dewatering unit and for better evaluation of system performance that meets the design criteria and future requirements for sludge disposal.
\end{abstract}

Keyword: Centrifuge dewatering unit; Bio-solids; Principle component analysis; Multiple linear regression; Sludge; Wastewater treatment 